\title{
Aspectos populacionais de Serrapinnus notomelas (Eigenmann, 1915) e Bryconamericus stramineus Eigenmann, 1908 (Characiformes: Characidae) em riachos da bacia do rio Ivinhema, Alto Rio Paraná
}

\author{
Luzia da Silva Lourenço ${ }^{1,3}$, Yzel Rondon Súarez ${ }^{2}$ \& Alexandro Cezar Florentino ${ }^{1}$ \\ ${ }^{1}$ Laboratório de Ecologia e Manejo de Recursos Pesqueiros, Instituto de Biociências, \\ Universidade Federal de Mato Grosso - UFMT, \\ CEP 78060-900, Cuiabá, MT, Brazil, \\ e-mail: alexandrocezar@yahoo.com.br \\ ${ }^{2}$ Laboratório de Ecologia, Centro Integrado de Análise e Monitoramento Ambiental - CInAM, \\ Universidade Estadual de Mato Grosso do Sul - UEMS, \\ Rod.Dourados-Itahum, Km 12, CEP 79804-970, Dourados, MS, Brazil, e-mail: yzel@uems.br \\ ${ }^{3}$ Corresponding author: Luzia da Silva Lourenço, e-mail: bioluzia@yahoo.com.br
}

LOURENÇO, L.S., SÚAREZ, Y.R. \& FLORENTINO, A.C. 2008. Populational aspects of Serrapinnus notomelas (Eigenmann, 1915) e Bryconamericus stramineus Eigenmann, 1908 (Characiformes: Characidae) in streams of Ivinhema River Basin, Upper Paraná Basin. Biota Neotrop. 8(4): http://www.biotaneotropica.org.br/v8n4/ en/abstract?article+bn00408042008.

\begin{abstract}
The knowledge of population parameters for Neotropical fish species is a great challenge to ichthyology. In this paper we analyzed the weight/length relationship, parameters of growth, mortality, recruitment and evaluate the influence of seasonal variation of rainfall on the mean weight of individuals and the pattern of recruitment for two fish species in streams of the Ivinhema River Basin-MS. The samples were done monthly from January to December 2002, with standardized effort between the sites. The equation that describe the weight/length relationship for Serrapinnus notomelas is (weight $=0.0000191 *$ length standard ${ }^{3.106}$ ) and for Bryconamericus stramineus is (weight $=0.0000080 *$ length standard ${ }^{3.193}$ ), being the constant b significantly higher than three. The asymptotic length for $S$. notomelas was $42.1 \mathrm{~mm}$ and for $B$. stramineus was $54.7 \mathrm{~mm}$. Serrapinnus notomelas presented larger natural mortality $(\mathrm{Z}=1.08)$ than $B$. stramineus $(\mathrm{Z}=0.93)$ and higher growth rate $(S$. notomelas $\mathrm{k}=0.60$ and B. stramineus $\mathrm{k}=0.54)$; and $S$. notomelas presented a lower longevity (4.99 years) than $B$. stramineus (5.54 years). The Index of growth performance $(\phi)$ calculated for $S$. notomelas was 3.027, while for B. stramineus was 3.208. We verified significant seasonal variation in the adjusted mean weight for both studied species. The recruitment pattern for $S$. notomelas and $B$. stramineus also follows a seasonal pattern, with two recruitment picks in the year, and $S$. notomelas presented a recruitment pick in March and other in June, while B. stramineus presents a pick in April and other in October.

Keywords: ecology, fish, weight/length relationships, population parameters.
\end{abstract}

LOURENÇO, L.S., SÚAREZ, Y.R. \& FLORENTINO, A.C. 2008. Aspectos populacionais de Serrapinnus notomelas (Eigenmann, 1915) e Bryconamericus stramineus Eigenmann, 1908 (Characiformes: Characidae) em riachos da bacia do rio Ivinhema, Alto Rio Paraná. Biota Neotrop. 8(4): http://www.biotaneotropica.org. br/v8n4/pt/abstract?article+bn00408042008.

Resumo: O conhecimento dos parâmetros populacionais para as espécies de peixes neotropicais é um grande desafio da ictiologia. No presente trabalho analisamos a relação peso/comprimento, parâmetros de crescimento, mortalidade, recrutamento e avaliamos a influência da variação sazonal da precipitação sobre o peso médio dos indivíduos e o padrão de recrutamento para duas espécies de peixes em riachos da bacia do rio Ivinhema, Estado de Mato Grosso do Sul - Brasil. As amostragens foram realizadas mensalmente de janeiro a dezembro/2002, com esforço padronizado entre os locais. A equação que descreve a relação peso total/comprimento padrão para Serrapinnus notomelas é (Peso = 0,0000191* LS $\left.{ }^{3,106}\right)$ e para Bryconamericus stramineus é (Peso = 0,0000080* LS $\left.{ }^{3,193}\right)$, sendo a constante b significativamente maior que três. O comprimento assintótico para $S$. notomelas foi de $42,1 \mathrm{~mm}$ e para B. stramineus foi de $54,7 \mathrm{~mm}$. Serrapinnus notomelas apresentou maior mortalidade natural $(\mathrm{Z}=1,08)$ do que $B$. stramineus $(\mathrm{Z}=0,93)$ e maior taxa de crescimento ( $S$. notomelas $\mathrm{k}=0,60$ e $B$. stramineus $\mathrm{k}=0,54)$, sendo que $S$. notomelas apresentou uma menor longevidade (4,99 anos) do que B. stramineus (5,54 anos). O Índice de performance de crescimento $(\phi)$ calculado para $S$. notomelas foi de 3,027, enquanto para $B$. stramineus foi de 3,208. Constatamos variação sazonal significativa no peso médio ajustado para ambas as espécies estudadas. $\mathrm{O}$ padrão de recrutamento para $S$. notomelas e $B$. stramineus também segue um padrão sazonal, com dois picos de recrutamento no ano, sendo que $S$. notomelas apresentou um pico de recrutamento em março e outro em junho, enquanto B. stramineus apresentou um pico em abril e outro em outubro.

Palavras-chave: ecologia, peixes, relação peso/comprimento, parâmetros populacionais. 


\section{Introdução}

A ictiofauna neotropical é uma das mais diversificadas do mundo. Contudo, o conhecimento da biologia básica de suas espécies ainda é um dos maiores desafios da ictiologia (Lowe-McConnell 1999), sendo que as espécies de pequeno porte são as menos conhecidas, desde o ponto de vista taxonômico até a quantificação de aspectos da ecologia populacional, o que dificulta a adoção de medidas mais eficientes de manejo e conservação (Sanna-Kaisa \& Jukka 2004).

Desta forma, o conhecimento da estrutura populacional (eg. mudança da taxa de natalidade e mortalidade, fecundidade, padrão de recrutamento), além de fornecer informações sobre a entrada de novos indivíduos na população e da longevidade dos mesmos (LoweMcConnell 1999), permite a compreensão da influência de fatores ambientais, tais como temperatura, oxigênio dissolvido, salinidade, fotoperíodo e hidroperíodo e dos fatores bióticos como a competição e a predação (Wootton 1999) sobre a dinâmica populacional das espécies de peixes.

Diversos autores têm estudado espécies de pequeno porte na planície de inundação do Alto Rio Paraná (Braga \& Gennari-Filho 1990, Garutti \& Figueiredo-Garutti 1992, Giamas et al. 1992, Benedito-Cecilio et al. 1997, Lizama \& Ambrósio 1999, 2002, Piana et al. 2006). No entanto, poucos trabalhos foram realizados em riachos com espécies de pequeno porte, ainda que para as espécies Serrapinnus notomelas (Eigenmann 1915) e Bryconamericus stramineus Eigenmann 1908 destacam-se alguns trabalhos, tais como os de Lizama \& Ambrósio (1999, 2002) sobre a relação peso/comprimento e fator de condição para $S$. notomelas na planície alagável do rio Paraná, bem como o trabalho de Galuch et al. (2003) sobre o desenvolvimento inicial e distribuição espacial de B. stramineus e o trabalho de Piana et al. (2006) quantificando a importância de características bióticas e abióticas sobre a densidade populacional de $S$. notomelas, todos realizados na planície de inundação do rio Paraná.

Diante do exposto o presente estudo tem como objetivo fornecer informações sobre a estrutura em comprimento, a relação peso total/ comprimento, parâmetros de crescimento, mortalidade e bem como analisar a influência da variação sazonal da precipitação sobre o peso médio dos indivíduos e o padrão de recrutamento.

\section{Material e Métodos}

As coletas foram realizadas mensalmente em sete riachos da bacia do rio Ivinhema, Alto Rio Paraná (Figura 1) entre janeiro e dezembro de 2002. As amostragens foram realizadas com uma peneira retangular de armação metálica $(1,2 \times 0,8 \mathrm{~m})$ confeccionada com tela de mosquiteiro ( $2 \mathrm{~mm}$ de abertura de malha), sendo realizados 20 lances por local. Todos os riachos foram amostrados no período diurno em um trecho de aproximadamente $50 \mathrm{~m}$.

Em campo, os peixes foram fixados em formol a $10 \%$ e, posteriormente, os exemplares levados ao laboratório foram transferidos para álcool $70 \%$ para preservação e para obtenção do peso em balança analítica e do comprimento padrão, utilizando paquímetro com aproximação a $0,1 \mathrm{~mm}$.

A relação peso total/comprimento padrão para B. stramineus e $S$. notomelas foi obtida pelo ajuste de uma regressão não-linear, bem como o intervalo de confiança para o coeficiente angular "b" da regressão.

O comprimento assintótico foi estimado a partir do maior indivíduo capturado utilizando a equação de Pauly (1983):

$$
\mathrm{L} \infty=\mathrm{L}_{\max } / 0,95
$$

O valor estimado da taxa de crescimento (k) foi obtido utilizandose o método ELEFAN I (Eletronic Lengths-Frequency Analysis) (Pauly \& David 1981), inserido no programa FISAT.

$\mathrm{O}$ índice de performance de crescimento $(\phi)$ foi obtido para as duas espécies através da equação proposta por Pauly \& Munro (1984):

$$
\phi=\log \mathrm{k}+2 \log \mathrm{L} \infty
$$

Enquanto a longevidade foi estimada segundo a equação proposta por Taylor (1958):

$$
\mathrm{t} \max =\text { to }+2,996 / \mathrm{k}
$$

A mortalidade total $(\mathrm{Z})$, aqui definida como igual à mortalidade natural (M), foi obtida segundo a fórmula empírica de Pauly (1980),

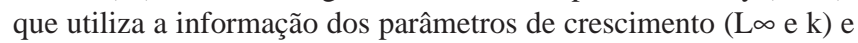
a temperatura média $\left({ }^{\circ} \mathrm{C}\right)$ do ambiente em que a espécie foi amostrada:

$$
\ln \mathrm{M}=-0,0152-0,279 \ln \mathrm{L} \infty+0,6543 \ln \mathrm{k}+0,463 \ln \mathrm{T}^{\circ} \mathrm{C} .
$$

Com o objetivo de analisar a influência da variação temporal sobre o peso dos indivíduos, para cada uma das espécies foi realizada uma análise de co-variância do peso (variável resposta) em função do mês da amostragem (variável explanatória) e do comprimento padrão (co-variável). Os dados de peso total e comprimento padrão foram previamente transformados utilizando $\log _{10}$. Adicionalmente, o peso médio ajustado para as duas espécies e a pluviosidade de um mês anterior foram correlacionados utilizando a correlação de Spearman.

O padrão de recrutamento foi obtido através da distribuição de freqüência de comprimento padrão e dos parâmetros de crescimento (Lo e k) estimados para cada espécie, utilizando-se a rotina incluída no FISAT (Gayanilo \& Pauly 1997). Posteriormente, foram relacionados os dados de recrutamento de ambas as espécies com a pluviosidade de um mês anterior utilizando a correlação de Spearman.

\section{Resultados}

Foram obtidos dados de peso e comprimento de 368 indivíduos de Serrapinnus notomelas e 198 indivíduos de Bryconamericus stramineus. O comprimento padrão médio de $S$. notomelas foi 23,2 $\mathrm{mm}(\mathrm{dp}=5,74)$, variando entre 9 e $40 \mathrm{~mm}$, enquanto para $B$. stramineus o comprimento padrão médio foi de $31,2 \mathrm{~mm}$ $(\mathrm{dp}=9,48)$, variando entre 9,6 e $52 \mathrm{~mm}$. Para $B$. stramineus constatamos, através da inspeção visual do histograma do comprimento padrão, que a população estudada apresentou quatro modas bem definidas (Figura 2).

O modelo de regressão não-linear gerado para a variação do peso em função do comprimento padrão para S. notomelas (Peso $=0,0000191 * \mathrm{LS}^{3,082}$ ) permitiu explicar 96\% da variação nos dados. Para B. stramineus o modelo gerado (Peso $=0,0000080 * \mathrm{LS}^{3,198}$ ) permitiu explicar 95,7\% da variação nos dados (Tabela 1 e Figura 3). Para as duas espécies o valor do intervalo de confiança do coeficiente angular "b" ajustado através da regressão não-linear foi estatisticamente maior que 3 , Sendo $(3,009-3,155)$ para $S$. notomelas e $(3,079-3,318)$ para B. stramineus, desta forma elas apresentam crescimento alométrico positivo.

Através da comparação dos intervalos de confiança do coeficiente angular da relação peso/comprimento constatamos que não existe diferença significativa na taxa de incremento de peso em função do comprimento. Contudo, em nossas amostragens, o comprimento má- 


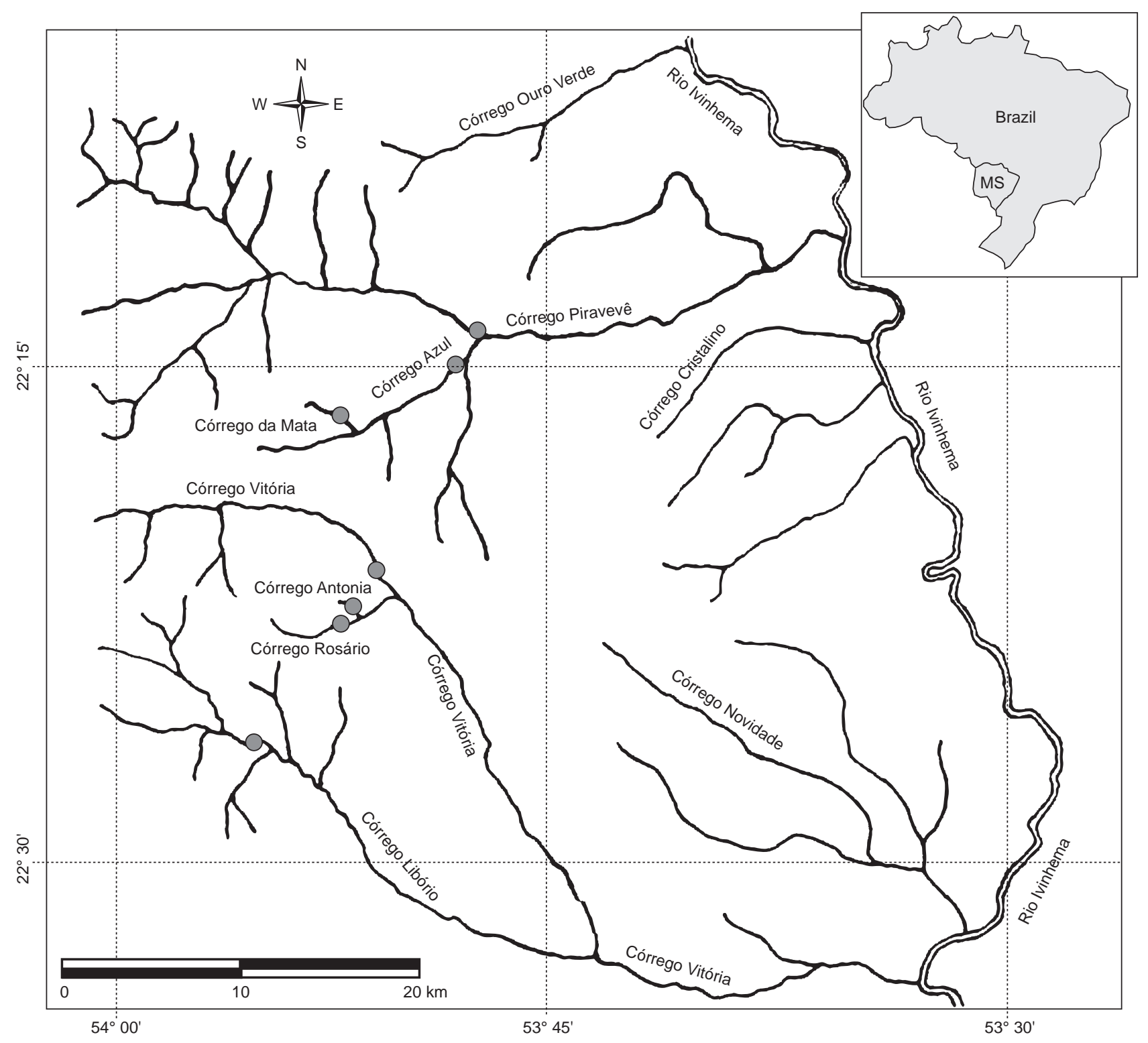

Figura 1. Localização dos riachos na bacia do rio Ivinhema-MS, Alto Paraná.

Figure 1. Location of the streams in the basin of the river Ivinhema-MS, Upper Parana.

ximo alcançado pelas espécies difere, sendo que $B$. stramineus é maior $\left(1_{\max }=52 \mathrm{~mm}\right)$ quando comparado com $S$. notomelas $\left(1_{\max }=40 \mathrm{~mm}\right)$, fato que influencia o coeficiente angular.

Estimamos o comprimento assintótico para $S$. notomelas em 42,1 mm, enquanto para B. stramineus o comprimento assintótico estimado foi de $54,7 \mathrm{~mm}$. Serrapinnus notomelas apresenta maior mortalidade natural $(Z=1,08)$ que $B$. stramineus $(Z=0,93)$ e maior taxa de crescimento ( $S$. notomelas $\mathrm{k}=0,60$ e $B$. stramineus $\mathrm{k}=0,54) ; S$. notomelas apresenta menor longevidade (4,99 anos) que $B$. stramineus (5,54 anos). O Índice de performance de crescimento $(\phi)$ calculado para $S$. notomelas foi de 3,027 , enquanto para B. stramineus foi de 3,208.

Utilizando a análise de co-variância constatamos que existe influência significativa da variação sazonal, interagindo com o comprimento padrão sobre o peso de ambas as espécies estudadas (Tabela 2), sendo que o peso médio ajustado para $S$. notomelas acompanha a mudança na pluviosidade ao longo do ano $(\mathrm{r}=0,580$; $\mathrm{p}=0,050$ ) (Figura 4), contudo, para B. stramineus esta correlação não foi significativa $(\mathrm{r}=0,545 ; \mathrm{p}=0,068)$.

O padrão de recrutamento para $S$. notomelas e $B$. stramineus também segue um padrão sazonal, com dois picos de recrutamento no ano, sendo que $S$. notomelas apresenta um pico de recrutamento em março e outro em junho, enquanto $B$. stramineus apresenta um pico em abril e outro em outubro (Figura 5), ou seja, nos meses com redução da pluviosidade. Ao correlacionar os dados de recrutamento e pluviosidade não evidenciamos relação para S. notomelas $(\mathrm{r}=-0,11$; $\mathrm{p}=0,732$ ), porém houve relação negativa entre a pluviosidade e recrutamento para $B$. stramineus $(\mathrm{r}=-0,580 ; \mathrm{p}=0,050)$.

\section{Discussão}

A estrutura em comprimento de uma população varia devido ao regime de recrutamento e mortalidade dos indivíduos. Tanto a popula- 
Tabela 1. Resultado da análise de regressão não-linear do peso em função do comprimento padrão (ls) dos indivíduos com os valores estimados de "a" e "b", bem como o intervalo de confiança, para os riachos da bacia do rio Ivinhema, MS no período de janeiro a dezembro/2002.

Table 1. Result of the analysis of non-linear regression of the weight in function of the standard length (1s) of the individuals with the estimated values of "a" and "b", as well as the confidence interval, for the streams of the basin of the river Ivinhema, MS in the period from January to December/2002.

\begin{tabular}{lccc}
\hline \multicolumn{1}{c}{ Espécie } & Constante a & Constante b & $\mathbf{r}^{2}$ \\
\hline Serrapinnus notomelas & $191 * \mathrm{e}-5(146 * \mathrm{e}-5$ a $235 * \mathrm{e}-5)$ & $3,082(3,009-3,155)$ & $0,960 * * *$ \\
Bryconamericus stramineus & $80 * \mathrm{e}-6(46 * \mathrm{e}-6$ a $113 * \mathrm{e}-5)$ & $3,198(3,079-3,318)$ & $0,957 * * *$ \\
\hline
\end{tabular}

*** = significativo a $\alpha=0,0001$

Tabela 2. Resultado da análise de co-variância do peso das espécies estudadas em função da variação sazonal e do comprimento padrão (ls) dos indivíduos para os riachos da bacia do rio Ivinhema, MS no período de janeiro a dezembro-2002.

Table 2. Result of the analysis of co-variance of the weight of the species studied in function of the seasonal variation and of the standard length (ls) of the individuals for the streams of the basin of the river Ivinhema, MS in the period of January the December-2002.

\begin{tabular}{ccccc}
\hline Espécie & $\mathbf{N}$ & $\mathbf{r}^{2}$ & F-meses & F-Comprimento padrão \\
\hline Serrapinnus notomelas & 368 & 0,907 & $7,346^{* * * *}$ & $3445,8^{* * *}$ \\
Bryconamericus stramineus & 198 & 0,970 & $4,817^{* * *}$ & $5188.4 * * *$ \\
\hline
\end{tabular}

*** = significativo a $\alpha<0,0001$
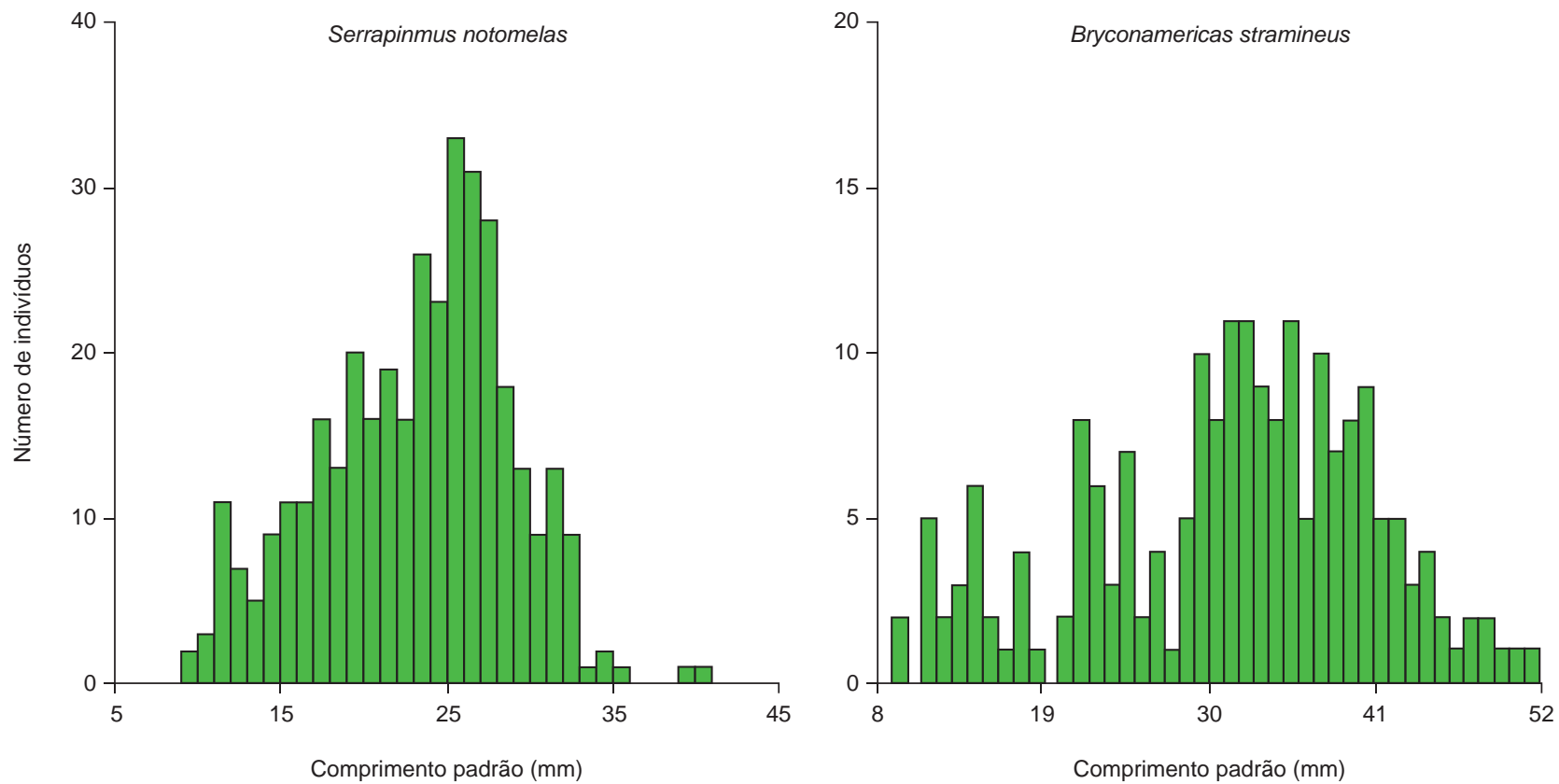

Figura 2. Histograma de comprimento padrão $(\mathrm{mm})$ para S. notomelas e B. stramineus nos riachos estudados na bacia do rio Ivinhema-MS.

Figure 2. Histogram of standard length ( $\mathrm{mm}$ ) for S. notomelas and B. stramineus in the streams studied in the basin of the river Ivinhema-MS.

ção de B. stramineus como a população de $S$. notomelas apresentaram um padrão polimodal na distribuição das classes de comprimento o que pode ser um indicativo de repetidos episódios reprodutivos ao longo do ano, e consequentemente a presença de várias coortes na população das duas espécies analisadas em riachos do rio Ivinhema. As alterações na estrutura em comprimento podem resultar do efeito das variáveis abióticas e bióticas na taxa de natalidade e sobrevivência de cada população (Gurgel 2004) e da variação dos atributos ambientais, que determina o estado nutricional da população (Bagarinão \& Thayaparam 1986). As diferenças encontradas por Orsi et al. (2004) na distribuição de comprimento da população de Astyanax altiparanae em trechos do rio Paranapanema, Paraná, resultou de diferenciadas estratégias de desenvolvimento, ou seja, épocas distintas de recrutamento nos trechos amostrados, onde tanto os fatores abióticos locais, como a predação, a disponibilidade de alimento determinaram a estrutura populacional da espécie.

Trexler \& Travis (1990) observaram na população de Poecilia latipinna que o efeito do ambiente não é forte o suficiente para levar a variações no comprimento corporal entre populações, e que as diferenças intrínsecas entre populações naturais, podem ser resultado da diferença na distribuição de frequiência de genótipos presentes nas populações.

Lizama \& Ambrósio (1999) analisando a relação peso/comprimento para nove espécies de peixes na planície de inundação da bacia do rio Paraná encontraram para S. notomelas valores para a 

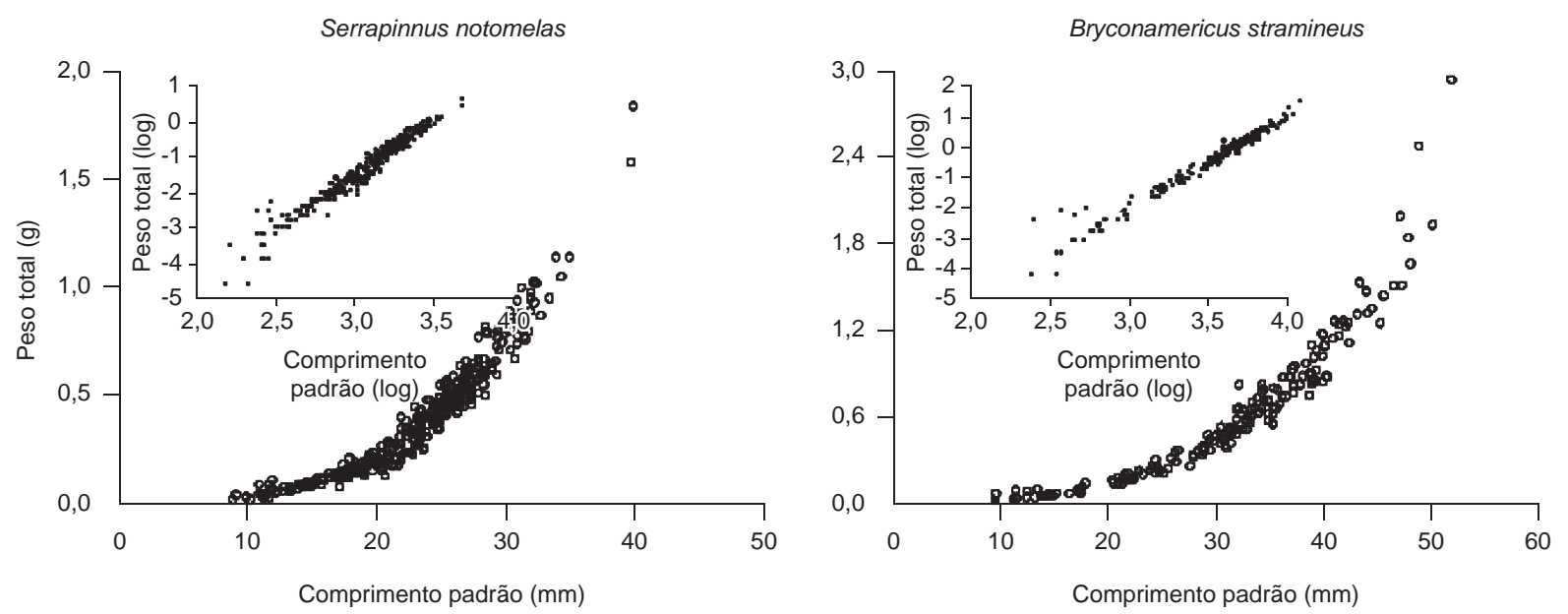

Figura 3. Relação Peso/Comprimento para S. notomelas e B. stramineus nos riachos estudados da bacia do rio Ivinhema-MS.

Figure 3. Relationship weight/length for S. notomelas and B. stramineus in the studied streams of the basin of the river Ivinhema-MS.

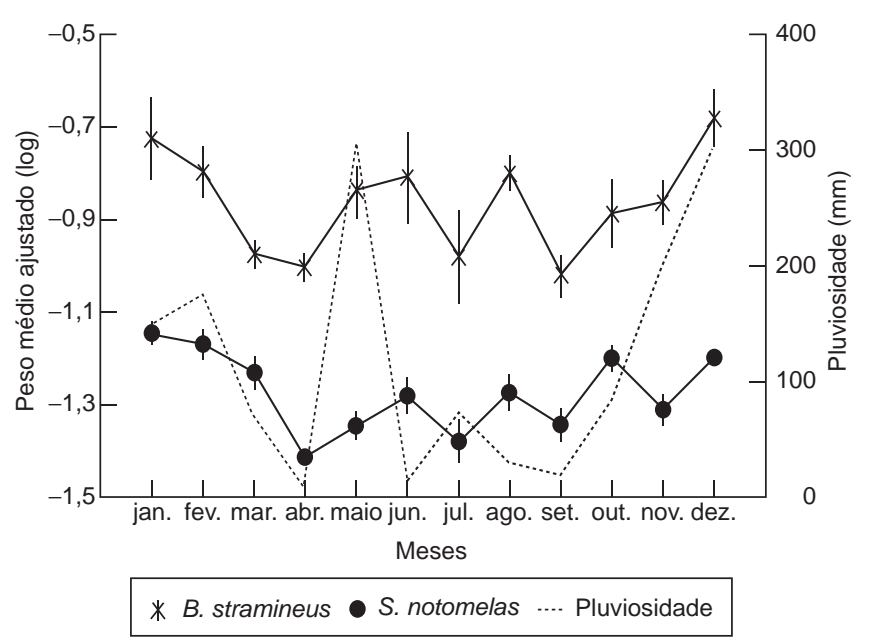

Figura 4. Variação sazonal na pluviosidade, peso total médio ajustado $\left(\log _{10}\right) \pm$ erro padrão para $B$. stramineus e $S$. notomelas nos riachos estudados da bacia do rio Ivinhema-MS.

Figure 4. Seasonal variation in the rainfall, weight medium total fitting $\left(\log _{10}\right)$ \pm standard mistake for $B$. stramineus and $S$. notomelas in the studied streams of the basin of the river Ivinhema-MS.

constante "a" bem acima $(0,0199)$ dos encontrados no presente trabalho (0,0000191), ainda que o coeficiente angular da regressão "b" tenha sido similar $(3,09)$ ao encontrado no presente estudo $(3,106)$. Desta forma duas hipóteses podem explicar a diferença encontrada no parâmetro "a" entre o trabalho de Lizama \& Ambrósio (1999) e o presente trabalho: 1) O fato das amostragens realizadas por estas autoras terem sido desenvolvidas em um outro tipo de ambiente (planície de inundação) quando comparado ao presente estudo, sendo assim, a maior produtividade na planície de inundação poderia atuar levando ao nascimento de indivíduos mais pesados que nos riachos por nós estudados; 2) A grande dispersão dos dados de peso na população estudada por estas autoras teriam conduzido a uma falha na estimativa do parâmetro a.

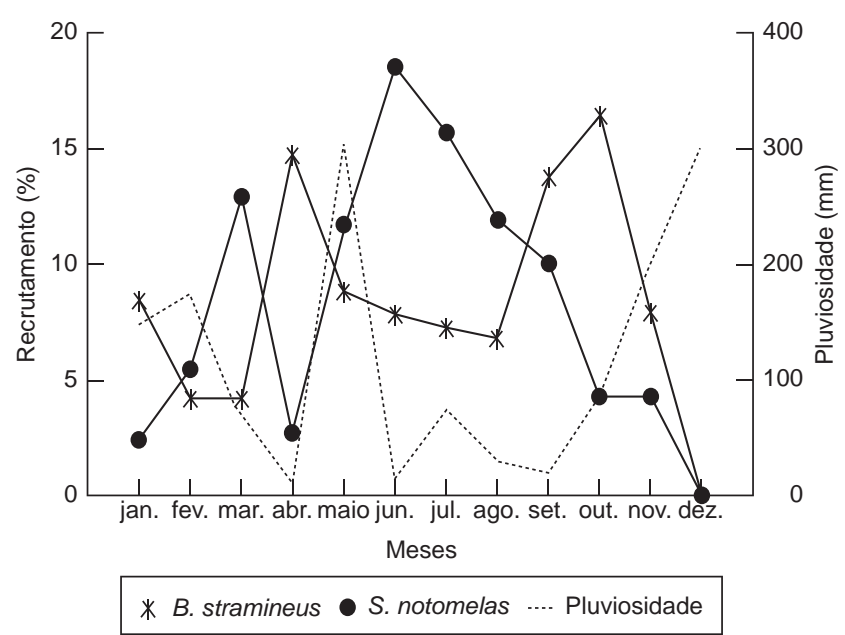

Figura 5. Padrão de recrutamento de $S$. notomelas e B. stramineus em riachos da bacia do rio Ivinhema-MS.

Figure 5. Pattern of recruitment of $S$. notomelas and B. stramineus in streams of the basin of the river Ivinhema-MS.

No presente estudo, constatamos que S. notomelas apresenta maior peso ao nascer que $B$. stramineus, e que ambas apresentam o coeficiente angular da regressão estatisticamente iguais (sobreposição dos intervalos de confiança), sendo que, ambas as espécies apresentam crescimento alométrico positivo. Giarrizzo et al. (2006) também encontraram similaridade na forma de crescimento de espécies pertencentes à mesma família como no presente estudo.

O desempenho de crescimento, ou seja, o incremento em peso e comprimento de espécies distintas pode ser semelhante, porque ao nascerem os indivíduos investem em crescimento corporal, e quando atingem a maturidade sexual a energia adquirida é acumulada e destinada aos eventos reprodutivos (Costa \& Araújo 2003). Ambas as espécies são presas, portanto pode ser esta, outra explicação para 
a similar forma de desenvolvimento exibida por $S$. notomelas e $B$. stramineus, pois quanto mais rápido elas atingem o comprimento máximo teórico maiores são as chances de sobrevivência, uma vez que os indivíduos menores são mais vulneráveis ao processo de predação (Reznick et al. 1996).

Quanto aos parâmetros de crescimento (Lo e K) espera-se que eles sejam diferentes entre espécies. No entanto, apesar dos parâmetros divergirem, as espécies estudadas seguiram um padrão predito de crescimento, pois $S$. notomelas alcançou o menor comprimento máximo teórico e a maior taxa de crescimento, e em conseqüência uma maior taxa de mortalidade natural do que B. stramineus. Deste modo, quanto menor o comprimento assintótico da espécie, maior é a taxa de crescimento, sendo que em função do pequeno comprimento corporal, maiores são as chances de serem predadas ou eliminadas do ambiente em condições adversas, o que resultaria, por conseguinte, em uma menor longevidade e elevada taxa de mortalidade.

Quando o crescimento em comprimento é descrito pelo mesmo modelo pode se comparar o desempenho de crescimento entre espécies de formas similares (Mateus \& Penha 2008), sendo que a ocorrência de ampla variação nos valores do desempenho de crescimento pode ser um indicativo de erro na estimativa de $(\phi)$. No entanto, o cálculo de $(\phi)$ para S. notomelas e B. stramineus ficou em torno de três, assim como para as espécies de Roeboides paranensis $(\phi=3,64)$ (Capistrano-Santana et al. 2004) e Moenkhausia dichroura $(\phi=3,75)$ (Cunha et al. 2007), ambos na baía da Onça, uma lagoa perene localizada à margem do rio Aquidauana - MS. Portanto, esses resultados sugerem uma satisfatória estimativa dos parâmetros de crescimento, pois para espécies de uma mesma família este índice não deve variar muito.

Para ambas as espécies, o comprimento padrão interagiu com a variação sazonal na determinação do peso dos indivíduos, o que associado à concordância entre a variação na pluviosidade e o peso médio ajustado das espécies sugere que as variações no nível da água são fatores de grande importância na dinâmica populacional destas espécies. Conclui-se que o melhor bem estar dos indivíduos ocorreu na estação da chuva, quando a condição do habitat torna-se favorável para o desenvolvimento do indivíduo, devido ao aumento na disponibilidade de abrigos e alimentos (Lowe-McConnell 1999, Bravo et al. 2001, Winemiller \& Dailey 2002, Silvano et al. 2003).

As populações de peixes exibem certa plasticidade e/ou adaptação às condições locais do habitat. Portanto o efeito de algumas variáveis ambientais sobre os parâmetros populacionais pode variar dependendo dos atributos ambientais locais. McManus \& Travis (1998) avaliaram o efeito da temperatura e da salinidade na biomassa corporal de machos de Poecilia latipinna em quatro populações do Norte da Flórida, e observaram uma ampla variação no peso dos indivíduos ao atingirem a maturidade, na análise não houve efeito da salinidade, mas sim interação entre população e temperatura, indicando que o peso dos machos foi alterado pela mudança de temperatura, com a magnitude do efeito variando entre machos de diferentes locais.

A relação entre a pluviosidade e o peso médio de $S$. notomelas, sugere que esta espécie seja mais dependente da sazonalidade das chuvas, ou até mesmo da variação da temperatura da água, principalmente quando consideramos seu menor tamanho médio em relação a B. stramineus. Assim, diferenças no crescimento podem estar relacionadas à variação sazonal na temperatura da água, como sugerido por De Graaf (2003) estudando o crescimento de peixes na planície de inundação de Bangladesh. Assim como por Trexler et al. (1990) ao avaliar o crescimento de fêmeas de Poecilia latipina na Florida. Conduto de acordo com Trexler \& Travis (1990) a variação na salinidade também produz efeitos consideráveis sobre o crescimento de peixes na Flórida.
Para ambas as espécies analisadas neste trabalho o recrutamento ocorreu durante todo o ano, porém não ocorre relação entre a pluviosidade e recrutamento para a população de $S$. notomelas, contudo para a população de B. stramineus evidencia-se uma relação negativa, pois nos meses de aumento das chuvas na região ocorre um decréscimo no recrutamento, isto fica evidente no mês de dezembro. Lizama \& Ambrósio (2003) evidenciaram que o recrutamento de Moenkhausia intermedia é contínuo e presente durante todo o ano, na planície de inundação do rio Paraná, embora ocorram períodos de maiores intensidades. Este padrão de recrutamento observado é característico de espécies de pequeno porte que habitam ambientes de água doce em regiões tropicais. Além disso, os picos de recrutamento na sua grande maioria coincidem com o período de mudança no nível da água. Entretanto, Cunha et al. (2007), ao analisarem o padrão de recrutamento de Moenkhausia dichroura na Baía da Onça, uma lagoa do Pantanal do rio Aquidauana, MS, observaram que a entrada de indivíduos na população ocorreu uma vez no ano (outubro). A população de Astyanax janeiroensis no rio Ubatuba, Rio de Janeiro, praticamente se reproduziu ao longo de todo o ano com exceção de maio a junho, com maior intensidade reprodutiva entre novembro e março (Mazzoni et al. 2005). Mazzoni \& Silva (2006) evidenciaram exemplares de Bryconamericus microcephalus em processo reprodutivo entre junho e fevereiro, com pico de reprodução entre setembro e dezembro em um riacho costeiro da Mata Atlântica, Ilha Grande, Rio de Janeiro. Lampert et al. (2007) constataram que B. stramineus possui desova parcelada com época de reprodução ocorrendo entre setembro e dezembro, com um pico menor em fevereiro no rio Ibicuí, RS. Neste contexto, a forma como as fêmeas liberam seus ovócitos dentro de um período reprodutivo, definido como tipo de desova (Vazzoler 1996), representa uma vantagem, uma vez que pode aumentar o sucesso reprodutivo, especialmente para as espécies de pequeno porte incapazes de estocar uma grande quantidade de ovócitos (Lampert et al. 2007). Este comportamento explicaria também a existência de dois picos de recrutamento observada no presente estudo, que seria então, resultado de mais de um evento reprodutivo ao longo do ano.

As espécies acima citadas apresentam uma mesma estratégia reprodutiva que é geneticamente determinada pela história de vida ou pelo comportamento programado. As táticas (estágios ontogenéticos ou ações específicas), que compõem a estratégia, podem diferir entre ambientes para que cada espécie ou população garanta que novos indivíduos sejam recrutados (Wootton 1999).

Em síntese conclui-se que ambas as espécies investem primeiro em incremento corporal, e que o peso relaciona-se positivamente com a pluviosidade local para $S$. notomelas, porém não há efeito significativo para $B$. stramineus, com vários pulsos de recrutamento ao longo do ano para ambas as espécies. Entretanto, embora nossos resultados e alguns estudos tenham evidenciado o efeito da sazonalidade do ambiente determinando as mudanças nos parâmetros populacionais, não podemos desconsiderar o efeito da densidade populacional que conduz a competição por recursos influenciando processos populacionais em ambientes naturais. Contudo, como nosso trabalho não objetivou avaliar esta abordagem sugerimos que estudos em ambientes com diferenças de densidade e de oferta de recursos poderiam esclarecer melhor sua importância sobre a dinâmica populacional destas espécies.

\section{Agradecimentos}

À Universidade Estadual do Mato Grosso do Sul/UEMS pelo apoio financeiro. Suely A. D. Molina, Amal Farhat, Priscilla Gobi pelo auxílio nos trabalhos de campo. Aos revisores pelas críticas e sugestões. 


\section{Referências Bibliográficas}

BAGARINÃO, T. \& THAYAPARAN, K. 1986. The length-weight relationship, food habitats and condition factor of wild juvenile milkfish in Sri Lanka. Aquaculture 55(3):241-246.

BENEDITO-CECILIO, E., AGOSTINHO, A.A., CARNELÓS-MACHADO VELHO, R.C. 1997. Length-weight relationship of fishes caught in the Itaipu reservoir Paraná, Brasil. Naga, The ICLARM Quaterly 20(3-4):57-61.

BRAGA, F.M.S. \& GENNARI-FILHO, O. 1990. Contribuição para o conhecimento da reprodução de Moenkhausia intermedia (Characidae, Tetragonopterinae) na represa de Barra Bonita, rio Piracicaba, SP. Naturalia 15:171-188.

BRAVO, R., SORIGUER, M.C., VILLAR N. \& HERNANDO, J.A. 2001. The dynamics of fish populations in the Palancar stream, a small tributary of the river Guadalquivir, Spain. Acta Oecologica 22 (1):9-20.

CAPISTRANO-SANTANA, A.C., ALMEIDA, R.S., ÁVILA, R.W. \& CATELLA, A.C. 2004. Estimativa dos parâmetros de crescimento de Roeboides paranensis: diferentes respostas para uma mesma pergunta. In IV Simpóso sobre Recursos Naturais e Sócio-econômicos do Pantanal Sustentabilidade Regional. Embrapa Pantanal, Corumbá. (CD-ROM).

COSTA, M.R. \& ARAÚJO, F.G. 2003. Lenth- weigth relationship and condition factor of Micropogonias furnieri (Desmarest) (Percuformes, Sciaenidae) in the Sepetiba Bay, Rio de Janeiro State, Brazil. Revta Bras. Zool. 20(4):685-690.

CUNHA, N.L., CATELLA, A.C. \& KINAS, M.A. 2007. Growth parameters estimates for a small fish of the Pantanal, Brazil: Moenkhausia dichroura (Characiformes; Characidae). Braz. J. Biol. = Rev. Bras. Biol. 67(2):293-297.

DE GRAAF, G. 2003. The flood pulse and growth of floodplain fish in Bangladesh, Fisheries Manag. Ecol. 10(4):241-247.

GALUCH, A.V., SUIBERTO, M.R., NAKATANI, K., BIALETZKI, A. \& BAUMGARTNER, G. 2003. Desenvolvimento inicial e distribuição temporal de larvas e juvenis de Bryconamericus stramineus Eigenmann, 1908 (Osteichthyes, Characidae) na planície alagável do alto rio Paraná, Brasil. Acta Sci. Biol. Sci. 25(2):335-343.

GIARRIZZO, T., JESUS, T.A.J.S., LAMEIRA, E.C., ALMEIDA, J.B.A., ISAAC, V. \& SAINT-PAUL, U. 2006. Weight-length relationships for intertidal fish fauna in a mangrove estuary in Northern Brazil. Appl. Ichthyol. 22(4):325-327.

GARUTTI, V. \& FIGUEIREDO-GARUTTI, M.L. 1992. Caracterização de populações do lambari Astyanax bimaculatus (Pisces, Characidae) procedentes do campus de Jaboticabal, UNESP, SP. Naturalia 17(1):17-29.

GAYANILO-JUNIOR, F.C. \& PAULY, D. 1997. The FAO-ICLARM Stock Assessment Tools (FISAT) Reference manual. FAO Computerized Information Series (Fisheries) 8:1-196.

GIAMAS, M.T.D., SANTOS, R.A., VERMULM JUNIOR, H., CAMPOS, E.C \& CAMARA, J.J.C. 1992. Determinação da curva de crescimento através da lepidologia em diferentes áreas do corpo de Astyanax bimaculatus (Linnaeus, 1758) (Pisces, characidae), na Represa de Ibitinga, SP. Braz. J. Vet. Res. Anim. Sci. 29(2):185-192.

GURGEL, H.C.B. 2004. Estrutura populacional e época de reprodução de Astyanax fasciatus (Curvier) (Characidae, Tetragonopterinae) do Rio Ceará Mirim, Poço Branco, Rio Grande do Norte, Brasil. Revta Bras. Zool. 21(1):131-135.

LAMPERT, V.R., AZEVEDO, M.A. \& FIALHO, C.B. 2007. Reproductive biology of Bryconamericus stramineus Eigenmann, 1908 (Ostariophysi: Characidae) from the Rio Ibicuí, RS, Brazil. Braz. Arch. Biol. Technol. 50(6):995-1004.

LIZAMA, M.A.P. \& AMBRÓSIO, A.M. 1999. Relação peso-comprimento e estrutura da população de nove espécies da família Characidae na planície de inundação do alto rio Paraná, Brasil. Rev. Bras. Zool. 16(3):779-788.

LIZAMA, M.A.P. \& AMBRÓSIO, A.M. 2002. Condition factor in nine species of fish of the Characidae family in the upper Paraná River floodplain. Braz. J. Biol. = Rev. Bras. Biol. 62(1):113-124.

LIZAMA, M.A.P. \& AMBRÓSIO, A.M. 2003. Crescimento, recrutamento e mortalidade do pequi Moenkhausia intermedia (Osteichthyes, Characidae) na planície de inundação do alto rio Paraná, Brasil. Acta Sci. Biol. Sci. 25(2):328-333.

LOWE-MCCONNELL, R.H. 1999. Estudos ecológicos de comunidades de peixes tropicais. EDUSP, São Paulo.

MATEUS, L.A.F. \& PENHA, J.M.F. 2007. Dinâmica populacional de quatro espécies de grandes bagres na bacia do rio Cuiabá, Pantanal norte, Brasil (Siluriformes, Pimelodidae). Revta Bras. Zool. 24(1):87-98.

MAZZONI, R. \& SILVA, A.P.F. 2006. Aspectos de história de vida de Bryconamericus microcephalus (Miranda Ribeiro) (Characiformes, Characidae) de um riacho costeiro de Mata Atlântica, Ilha Grande, Rio de Janeiro, Brasil. Rev. Bras. Zool. 23(1):228-233.

MAZZONI, R., MENDONÇA, R.S. \& CARAMASCHI, E.P. 2005 Reproductive Biology of Astyanax Janeiroensis (Osteichthyes, Characidae) from the Ubatiba river, Maricá, RJ, Brazil. Braz. J. Biol. = Rev. Bras. Biol. 65(4):643-649.

MCMANUS, M.G. \& TRAVIS, J. 1998. Effects of temperature and salinity on the life history of the sailfin molly (Pisces: Poeciliidae): lipid storage and reproduction allocation. Oecologia 114(3):317-325.

ORSI, M.L., CARVALHO, E.D. \& FORESTI, F. 2004. Biologia Populacional de Astyanax altiparanae Garutti \& Britski (Teleostei, Characidae) do Médio Rio Paranapanema, Paraná, Brasil. Rev. Bras. Zool. 21(2):207-218.

PAULY, D. \& DAVID, N. 1981. ELEFAN I, a BASIC programme for the objective extraction of growth parameters from length frequencies data. Meeresforschung. 28(4):205-211.

PAULY, D. \& MUNRO, J.L. 1984. Once more on the comparison of growth in fish and invertebrates. ICLARM Fishbyte 2(1):1-21.

PAULY, D. 1980. On the interrelationships between natural mortality, growth parameters and mean environmental temperature in 175 fish stock. International Council Exploration of the sea 39(2):175-192.

PAULY, D. 1983. Some simples methods for the assessment of tropical fish stocks. FAO Fisheries Technical Paper 234:1-52.

PIANA, P.A., GOMES, L.C., \& CORTEZ, E.M. 2006. Factors influencing Serrapinnus notomelas (Characiformes: Characidae) populations in upper Paraná river floodplain lagoons. Neotrop. Ichthyol. 4(1):81-86.

REZNICK, D.N., BUTLER, M.J., RODD, F.H. \& ROSS, P. 1996. Life-History Evolution in guppies (Poecilia reticulate) 6. Differential Mortality as a Mechanism for natural Selection. Evolution 50(4):1651-1660.

SANNA-KAISA, J. \& JUKKA, S. 2004. Sustentable use of ornamental fish populations in Peruvian Amazonia. Lyonia 7(2):53-59.

SILVANO, J., OLIVEIRA, C.L.C., FIALHO, C.B. \& GURGEL, H.C.B. 2003. Reproductive period and fecundity of Serrapinus piaba (Characidae: Cheirodontidae) from the rio Ceará Mirim, Rio Grande do Norte, Brasil. Neotrop. Ichthyol. 1(1):61-66.

TAYLOR, C.C. 1958. Cod growth and temperature. J. Cons. Int. Explor. Mer. 23:366-370.

TREXLER, J.C. \& TRAVIS, J. 1990. Phenotypic plasticity in the sailfin Molly, Poecilia latipina (Pisces: Poeciliidae). I. Field experiments. Evolution 44(1):143-156.

TREXLER, J.C., TRAVIS, J. \& TREXLER, M. 1990. Phenotypic plasticity in the sailfin Molly, Poecilia latipina (Pisces: Poeciliidae). II. Field experiments. Evolution 44(1):157-167.

VAZZOLER, A.E.A.M. 1996. Biologia da Reprodução de Peixes Teleósteos: Teoria e Prática. EDUEM, Maringá.

WINEMILLER, K.O. \& DAILEY, W.D. 2002. Life History Strategies, Population Dynamics, And Consequences For Supplemental Stocking Of Tarpon. Cont. Marine Sci. 35: 81-94.

WOOTTON, R.J. 1999. Ecology of Teleost Fishes. 2 ed. Chapman \& Hall, London. 